\title{
Photophysical Characterization of a Photochromic System: The Case of Merocyanine
}

540

\author{
Pedro F. Aramendia,* \\ Departamento de Quimica Inorgänica, Analitica y Quïmica Física, Facultad de Ciencias Exactas y Naturales, \\ $U B A$, Pabellón 2, C. Universitaria, 1428 Buenos Aires, Argentina
}

Ricardo Duchowicz, Lucia Scaffardi, and Jorge O. Tocho

Centro de Investigaciones Opticas, Casilla de Correo 124, 1900 La Plata, Argentina

(Received: November 10, 1988; In Final Form: June 29, 1989)

\begin{abstract}
The fluorescence emission of merocyanine 540 (MC540) in 95\% ethanol was studied under continuous irradiation. Fluorescence spectra from excited states of both normal $(N)$ and photoisomeric $(P)$ species are identical. The laser fluence dependence of the fluorescence intensity is interpreted on the basis of a photochromic system involving $\mathbf{N}$ and $\mathbf{P}$ ground states and first excited singlet states. Common flash photolysis equations are generalized in order to include a photoequilibrium between isomers. The emission data are used together with previous flash photolysis and optoacoustic results to obtain $\mathrm{P}$ fluorescence and photoisomerization quantum yields as $0.07 \pm 0.02$ and $0.20 \pm 0.04$, respectively, $P$ absorption cross section at the maximum $(560 \mathrm{~nm})$ as $4.74 \times 10^{-16} \mathrm{~cm}^{2}\left(125 \times 10^{3} \mathrm{M}^{-1} \cdot \mathrm{cm}^{-1}\right)$, and the energy difference between the ground states as $165 \mathrm{~kJ} \cdot \mathrm{mol}^{-1}$. Internal conversion, which can take place as a direct process or through a twisted state, is the main deactivation pathway for excited singlet $P$.
\end{abstract}

\section{Introduction}

Merocyanine 540 (MC540) is an anionic polymethine dye with various applications in photobiology. ${ }^{1}$ Since 1974 it has been

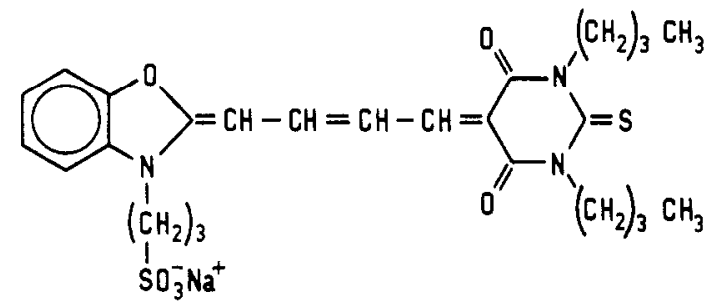

MC 540

used as a probe to monitor the state of membrane polarization through changes in its absorption or fluorescence properties. ${ }^{2}$ It was later employed to selectively stain and kill leukemic and immature hemopoietic cells upon irradiation. ${ }^{3}$ Finally its ability to sensitize the inactivation of encapsulated viruses was reported. ${ }^{4}$ In view of the multiple applications to photobiology, many studies were devoted to the interaction of MC540 with micelles, ${ }^{5}$ liposomes, ${ }^{6}$ and membranes. ${ }^{7}$

(1) For a recent review see: Sieber, F. Photochem. Photobiol. 1987, 46, 1035-1042.

(2) (a) Ross, W. N.; Salzberg, B. M.; Cohen, L. B.; Davila, H. V. Biophys. J. 1974, 14, 983. (b) Waggoner, A. J. Membr. Biol. 1976, 27, 317. (c) Waggoner, A. S.; Grinvald, A. Ann. N.Y. Acad. Sci. 1977, 303, 217. (d) Waggoner, A. S. Annu. Rev. Biophys. Bioeng. 1979, 8, 47. (e) Easton, T. G.; Valinsky, J. E.; Reich, E. Cell 1978, 13, 475.

(3) (a) Valinsky, J. E.: Easton, T. G.; Reich, E. Cell 1978, 13, 487. (b) Meagher, R. C.; Sieber, F.; Spivak, J. L.; J. Cellular Physiol. 1983, 116, 118. (c) Sieber, F.; Spivak, J. L.; Sutcliffe, A. M. Proc. Natl. Acad. Sci. USA 1984, 81, 7584. (d) Sieber, F.; Sieber-Blum, M. Cancer Res. 1986, 46, 4892. (4) Sieber, F.; O'Brien, J. M.; Krueger, G. J.; Schrober, S. L.; Burns, W. M.; Sharkis, S. J.; Sensenbrenner, L. L. Photochem. Photobiol. 1987, 46, 707-711.

(5) (a) Guarcello, V.; Stern, A.; Rizza, V. Biochim. Biophys. Acta 1987, 917, 318-323. (b) Dodin, G.; Aubard, J.; Falque, D. J. Phys. Chem. 1987, $91,1166-1172$.

(6) (a) Dragsten, P. R. Weeb, W. W. Biochemistry 1978, 17, 5228. (b) Lelkes, P. I.; Miller, I. R. J. Membr. Biol. 1980, 52, 1. (c) Lelkes, P. I.; Bach, D.; Miller, I. R. J. Membr. Biol. 1980, 54, 141. (d) Williamson, P.; Mattocks, K.; Schlengel, R. A. Biochim. Biophys. Acta 1983, 732, 387. (e) Verkman, A. S.; Frosch, M. P. Biochemistry 1985, 24, 7117. (f) Dodin, G.; Dupont, J. J. Phys. Chem 1987, 91, 6322. (g) Kalyanasaiam, B.; Feix, J. B.; Sieber, F.; Thomas, J. P.; Girotti, A. W. Proc. Natl. Acad. Sci. USA 1987, 84, 2999. (h) Verkman, A. S. Biochemistry 1987, 26, 4050.
Regarding the photophysical properties, the first results were related to the fluorescence characteristics in solution and micelles. ${ }^{8}$ Recently a fluorescence and photoisomerization study as well as the singlet molecular oxygen sensitization properties was reported ${ }^{9}$ and, finally, a detailed comparison of the photophysical properties in ethanol and in liposomes was published. ${ }^{10}$

In alcohols MC540 has a fluorescence quantum yield of ca. $20 \%$ at room temperature and a singlet lifetime of $400 \mathrm{ps.} \mathrm{It} \mathrm{has} \mathrm{a}$ very low triplet and singlet oxygen sensitization quantum yields (less than $1 \%$ and $0.1 \%$, respectively). Photoisomerization takes place from the first excited singlet state and is a very efficient process (ca. $50 \%$ yield). The absorption spectra of the stable ground state $(\mathrm{N})$ and the ground-state photoisomer $(\mathrm{P})$ strongly overlap., ${ }^{9} 10$ This later characteristic, together with the short singlet lifetime, makes the knowledge of the $P$ photophysics an important point to understand the behavior of the dye upon irradiation, even when short laser pulses are used. The last statement particularly points out the difficulties in the use of complete conversion method in flash photolysis, ${ }^{11}$ which was performed in ref 9 and 10 to determine the absorption coefficient of $P$ and further to calculate isomerization quantum yield. This method may produce misleading results if $\mathrm{P}$, present during the laser pulse, photochemically reverts to $\mathrm{N}$. In this case, the saturation limit would not reflect a complete conversion; rather $\mathrm{N}$ and $\mathrm{P}$ coexist in a photostationary state. In a previous work ${ }^{10}$ an upper limit was reported for the photoisomerization yield from flash photolysis measurements and a lower limit obtained from light-induced optoacoustics (LIOAS) results.

In this work, we perform steady-state fluorescence measurements, which have already proved to be a powerful tool for the characterization of 3,3'-diethyl oxadicarbocyanine iodide (DODCI) ${ }^{12}$ and we use the results together with flash photolysis and LIOAS early results ${ }^{10}$ to completely characterize $\mathrm{N}$ and $\mathrm{P}$ pho-

(7) See ref 6f and 6g. Humphries, G. M. K.; Lovejoy, J. P. Biochim Biophys. Res. Commun. 1983, 111, 768.

(8) Dixit, N. S.; Mackay, R. A. J. Am. Chem. Soc. 1983, 105, 2928.

(9) Hoebeke, M.; Seret, A.; Piette, J.; Van de Vorst, A. J. Photochem. Photobiol. B 1988, $1,437$.

(10) Aramendia, P. F.; Krieg, M.; Nitsch, C.; Bittersmann, E.; Braslavsky, S. E. Photochem. Photobiol. 1988, 48, 187

(11) Hug, G. L.; Carmichael, I. J. Photochem. 1985, 31, 179.

(12) Scaffardi, L.; Bilmes, G. M.; Schinca, D.; Tocho, J. O. Chem. Phys. Lett. 1987, 140,163. 


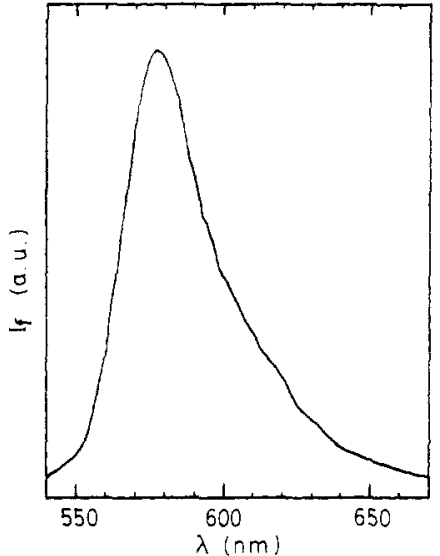

Figure 1. Uncorrected fluorescence emission spectrum of MC540 in $95 \%$ ethanol at excitation fluence $\left(I_{1}\right)=0.071 \mathrm{~W} \cdot \mathrm{cm}^{-2} . \lambda_{\text {exe }}$ was $514 \mathrm{~nm}$. No spectral distribution change is observed at $I_{1}=1.8 \mathrm{~kW} \cdot \mathrm{cm}^{-2}$.

tophysics in a system where both absorption spectra strongly overlap.

\section{Materials and Methods}

1. Chemicals. MC540 (Sigma, Deisenhofen, $\epsilon_{\max }(95 \% \mathrm{EtOH})$ $=1.65 \times 10^{5} \mathrm{M}^{-1} \cdot \mathrm{cm}^{-1}$ ) was used as received. Ethanol $95 \%$ was analytical grade. Samples were prepared immediately before use from a stock $(0.4 \mathrm{mM})$ solution.

2. Fluorescence Measurements. The light source was an argon ion laser (Spectra Physics 165) or an argon ion pumped dye laser (Spectra Physics 375). The light passed through a rotating half-wavelength plate, a polarizer, and a $0.48 \mathrm{~mm}$ diameter pinhole placed just in front of the sample holder. The sample was irradiated in a $1 \mathrm{~cm}^{2}$ fluorescence quartz cuvette and was pumped from a reservoir at a flux speed of $20 \mathrm{~mL} / \mathrm{s}$. A radiant power meter (Spectra Physics 404) monitored the laser power continuously during the measurements. Fluorescence was observed at $90^{\circ}$ relative to excitation. The laser beam impinged the cuvette very near to the observation side. The fluorescence light was gathered by a lens and passed either through a $633-\mathrm{nm}, 10 \mathrm{~nm}$ bandwidth, interference filter or a Jarrell-Ash Model 82-025 monochromator before it was detected by an R928 Hammamatsu photomultiplier tube. The signal was fed into a boxcar integrator (PAR 162) which was triggered internally at $1 \mathrm{kHz}$.

Fluorescence spectra were recorded by using the monochromator at different irradiation fluences or the fluorescence intensity was monitored as a function of excitation fluence by using the interference filter. In this arrangement, the laser power and the boxcar signal were plotted simultaneously in an $X, Y, Y^{\prime}$ recorder (Hewlett Packard). The laser power was varied by means of the electric current or by rotating the half-wavelength plate.

\section{Results}

The measurements were performed at $20 \pm 2{ }^{\circ} \mathrm{C}$. The absorbance of the MC540 solution in ethanol $95 \%$ was always less than 0.05 in $1 \mathrm{~cm}$ at the excitation wavelength. Typically it was $0.01 / \mathrm{cm}$.

The substance was excited at $488,514,580,590$, and $595 \mathrm{~nm}$. The excitation fluence $\left(I_{1}\right)$ was varied between the maximum possible and the minimum which produced a detectable fluorescence signal (typical variation range 3.5 orders of magnitude). In all cases, the maximum power was included in the range where an appreciable conversion to the photoisomer could be attained.

The MC540 fluorescence emission spectrum is shown in Figure 1. No spectral distribution change could be observed when the excitation intensity was changed by a factor of 25000 , indicating that $N$ and $P$ have also identical fluorescence emission spectra. In view of this result, the fluorescence intensity $\left(I_{f}\right)$ in a particular wavelength range is always proportional to the whole emission.

The value of the boxcar output voltage (proportional to $I_{\mathrm{f}}$ ), normalized to the excitation fluence, i.e., $I_{\mathrm{f}} / I_{1}$, is plotted against the velocity of light absorption $a=I_{\mid} \sigma_{\mathrm{N}}$ at different excitation

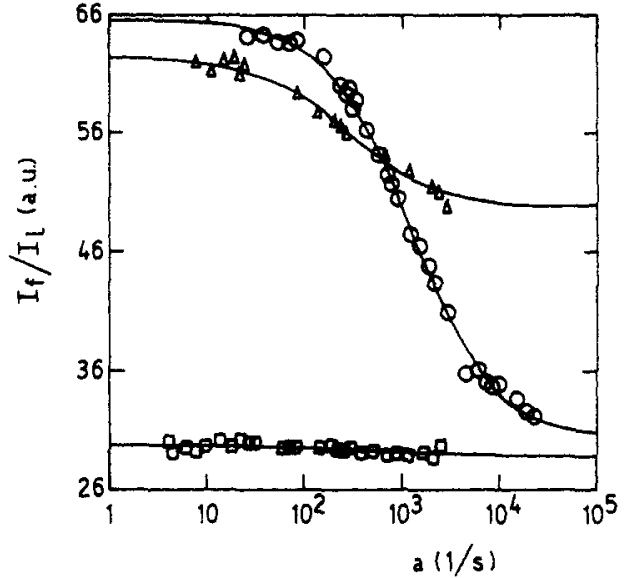

Figure 2. Plots of fluorescence intensity $\left(I_{t}\right)$ normalized to excitation fluence $\left(I_{1}\right)$, in arbitrary units, as a function of the rate constant of light absorption $\left(a=I_{\mathrm{L}} \sigma_{\mathrm{N}}=2.303 \epsilon_{\mathrm{N}} I_{\mathrm{L}}\right)$. The values of parameters that fit the experimental points are (O) $\lambda=514 \mathrm{~nm}, K=65.6 \pm 0.2, G=0.460$ \pm 0.005 , and $A_{\mathrm{s}}=(1220 \pm 55) \mathrm{s}^{-1} ;(\Delta) \lambda=580 \mathrm{~nm}, K=62.3 \pm 0.2$, $G=0.80 \pm 0.01$, and $A_{\mathrm{s}}=(270 \pm 11) \mathrm{s}^{-1}$; (口) $\lambda=595 \mathrm{~nm}, K=29.6$ $\pm 0.1, G=0.97 \pm 0.01$, and $A_{1}=(300 \pm 200) \mathrm{s}^{-1}$. See Discussion and eq 4 .

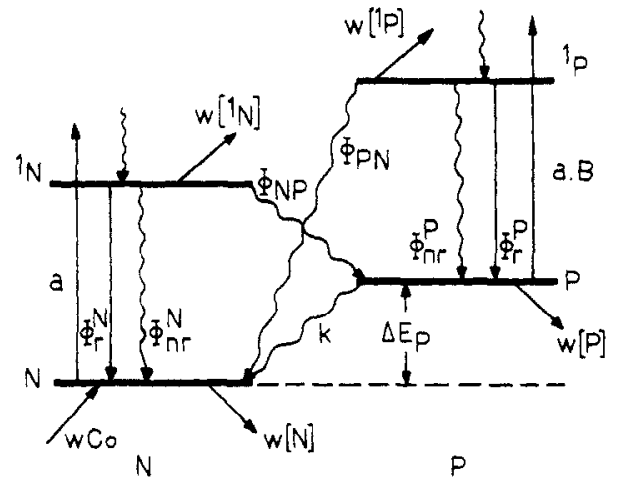

Figure 3. Four-level system used for the interpretation of the photochromic system. The activation-deactivation channels are indicated by rate constants $(a, B a, k)$ or quantum yields $\left(\Phi_{f}, \Phi_{\mathrm{NP}}, \Phi_{\mathrm{PN}}\right)$.

wavelengths in Figure 2. $\sigma_{\mathrm{N}}$ is the absorption cross section coefficient of $\mathrm{N}$ at the excitation wavelength.

In these plots the low excitation $a$-independent range corresponds to $\mathrm{N}$ fluorescence and the high, also $a$-independent range corresponds to the photoequilibrium between $\mathrm{P}$ and $\mathrm{N}$ after the saturation is reached.

\section{Discussion}

The system can be kinetically analyzed on the basis of the four-level scheme depicted in Figure 3. Under steady-state irradiation conditions and low absorption, the kinetic equations are

$$
\begin{aligned}
& \frac{\mathrm{d}[\mathrm{N}]}{\mathrm{d} t}= \\
& -(a+w)[\mathrm{N}]+\frac{\left(1-\Phi_{\mathrm{NP}}\right)}{\tau_{f}^{\mathrm{N}}}\left[{ }^{1} \mathrm{~N}\right]+\frac{\Phi_{\mathrm{PN}}}{\tau_{\mathrm{f}}^{\mathrm{P}}}\left[{ }^{1} \mathrm{P}\right]+k[\mathrm{P}]+w c_{0}=0 \\
& \frac{\mathrm{d}\left[{ }^{1} \mathrm{~N}\right]}{\mathrm{d} t}=a[\mathrm{~N}]-\left(\frac{1}{\tau_{\mathrm{f}}^{\mathrm{N}}}+w\right)\left[{ }^{1} \mathrm{~N}\right]=0 \\
& \frac{\mathrm{d}\left[{ }^{1} \mathrm{P}\right]}{\mathrm{d} t}=B a[\mathrm{P}]-\left(\frac{1}{\tau_{\mathrm{f}}^{\mathrm{P}}}+w\right)\left[{ }^{1} \mathrm{P}\right]=0 \\
& \frac{\mathrm{d}[\mathrm{P}]}{\mathrm{d} t}=\frac{\Phi_{\mathrm{NP}}}{\tau_{\mathrm{f}} \mathrm{N}}\left[{ }^{1} \mathrm{~N}\right]+\frac{\left(1-\Phi_{\mathrm{PN}}\right)}{\tau_{\mathrm{f}}^{\mathrm{P}}}\left[{ }^{1} \mathrm{P}\right]-(B a+k+w)[\mathrm{P}]=0
\end{aligned}
$$


TABLE I: Values of Photoisomerization Quantum Yield from $\mathbf{N}$ to $P$ $\left(\Phi_{\mathrm{NP}}\right)$ and from $\mathrm{P}$ to $\mathrm{N}\left(\Phi_{\mathrm{PN}}\right)$, Difference Absorption Coefficient $(\Delta \epsilon$ $\left.=\epsilon_{\mathrm{P}}-\epsilon_{\mathrm{N}}\right)$, and Absorption Coefficient of the Photoisomer $\left(\epsilon_{\mathrm{P}}\right)$ at 560 $\mathrm{nm}$ as a Function of $x$, Where $x$ Is the Fraction of the Concentration $c_{0}$ Which Is Present as $[\mathrm{P}]$ in the Saturation-Photostationary State ${ }^{a}$

\begin{tabular}{cccccc}
\hline$x$ & $\begin{array}{c}\Delta \epsilon(560 \mathrm{~nm}), \\
10^{3} \mathrm{M}^{-1} \cdot \mathrm{cm}^{-1}\end{array}$ & $\begin{array}{c}\epsilon_{\mathrm{P}}(560 \mathrm{~nm}), \\
10^{3} \mathbf{M}^{-1} \cdot \mathrm{cm}^{-1}\end{array}$ & $\boldsymbol{\Phi}_{\mathrm{NP}}$ & $\Phi_{\mathrm{PN}}$ & $\begin{array}{c}p= \\
\Phi_{\mathrm{PN}} / \boldsymbol{\Phi}_{\mathrm{NP}}\end{array}$ \\
\hline 1.0 & -30.1 & 135 & 0.54 & 0.000 & 0.00 \\
0.9 & -33.4 & 132 & 0.49 & 0.068 & 0.14 \\
0.8 & -37.6 & 127 & 0.43 & 0.140 & 0.33 \\
0.7 & -43.0 & 122 & 0.38 & 0.220 & 0.58 \\
0.6 & -50.2 & 115 & 0.32 & 0.320 & 1.00
\end{tabular}

${ }^{a}$ See eq $\mathrm{Al}$ and text following it.

Considering that the excited-state concentrations are much less than the ground states and neglecting the depopulation of ${ }^{1} \mathrm{~N}$ and 'P by circulation $\left(w \ll 1 / \tau_{f}^{N}, 1 / \tau_{f}^{P}\right)$, we arrive at the expression

$$
[\mathrm{P}]=\frac{a \Phi_{\mathrm{NP}}}{\left(\Phi_{\mathrm{NP}}+B \Phi_{\mathrm{PN}}\right) a+k+w} c_{0}
$$

where $B=\sigma_{\mathrm{P}} / \sigma_{\mathrm{N}}=\epsilon_{\mathrm{P}} / \epsilon_{\mathrm{N}}, \Phi_{\mathrm{NP}}$ and $\Phi_{\mathrm{PN}}$ are photoisomerization quantum yields from ${ }^{1} \mathrm{~N}$ to $\mathrm{P}$ and ${ }^{1} \mathrm{P}$ to $\mathrm{N}$ respectively, $c_{0}$ is the total concentration, and $w$ is the rate of change of concentration by circulation of the solution.

The total fluorescence intensity is

$$
I_{\mathrm{Tf}}=\Phi_{\mathrm{f}}^{\mathrm{N}} a[\mathrm{~N}]+\Phi_{\mathrm{f}}{ }^{\mathrm{P}} a B[\mathrm{P}]
$$

with $\Phi_{f}$, fluorescence quantum yields. If we replace (2) in (3) and $[\mathrm{P}]+[\mathrm{N}]=c_{0}$ is used, we obtain

$$
\frac{I_{\mathrm{f}}}{I_{\mathrm{l}}}=K\left(\frac{a_{\mathrm{s}}+a G}{a_{\mathrm{s}}+a}\right)
$$

where $K$ is a proportionality constant, $G=B(p+f) /(B p+1)$, $a_{\mathrm{s}}=(k+w) /\left(\Phi_{\mathrm{NP}}(B p+1)\right), p=\Phi_{\mathrm{PN}} / \Phi_{\mathrm{NP}}$, and $f=\Phi_{\mathrm{f}}^{\mathrm{P}} / \Phi_{\mathrm{f}} \mathrm{N}$. Equation 4 is used to fit the data of Figure 2. The fitting parameters are also quoted in the graphs. Equation 4 contains three adjustable parameters $K, a_{\mathrm{s}}$, and $G$. No useful information can be extracted from $K$, which depends on factors such as spectral bandwidth, photomultiplier sensitivity and applied voltage, and geometry. $a_{\mathrm{s}}$ is not meaningful either because $w$, the speed of sample renewal within the irradiated volume, is unknown due to turbulence in the flow. The useful information, regarding fluorescence and photoisomerization quantum yields of $\mathrm{N}$ and $\mathrm{P}$, is contained in $G$. This parameter is obtained from the measurements at different wavelengths, and it is used in the form

$$
G^{-1}=(p / p+f)+(1 / p+f) B^{-1}
$$

A plot of $G^{-1}$ vs $B^{-1}$ allows the calculation of $p$ and $f$. Nevertheless, this is not a straightforward process and an iterative method had to be used, because $B$ values are unknown. In order to know them, we need a further analysis of the flash photolysis data of ref 10 . In that work $\Phi_{\mathrm{NP}}$ was estimated to range between 0.54 and 0.32 . The upper limit corresponds to the assumption $\Phi_{\mathrm{PN}}=0$ and the lower corresponds to the maximum $\Phi_{\mathrm{PN}}$. In.the general case, we can write

$$
\Phi_{\mathrm{NP}}=\Phi_{\mathrm{NP}}{ }^{\max x}
$$

and for the difference absorption coefficient $\Delta \epsilon_{\lambda_{\alpha}}=\left(\epsilon_{\mathrm{P}}-\epsilon_{\mathrm{N}}\right)_{\lambda_{\alpha}}$,

$$
\Delta \epsilon_{\lambda_{\alpha}} x=-30 \times 10^{3} \mathrm{M}^{-1} \cdot \mathrm{cm}^{-1} \quad \text { at } \lambda_{\alpha}=560 \mathrm{~nm}
$$

$x$ is a factor that takes into account the back isomerization ${ }^{1} \mathrm{P}$ to $\mathrm{N}$ and is defined as

$$
x=\frac{\epsilon_{\mathrm{N}} \Phi_{\mathrm{NP}}}{\epsilon_{\mathrm{P}} \Phi_{\mathrm{PN}}+\epsilon_{\mathrm{N}} \Phi_{\mathrm{NP}}}
$$

Equations $6 \mathrm{a}$ and $6 \mathrm{~b}$ correspond to eq $\mathrm{A} 5$ and $\mathrm{A} 3$ of the Appendix and the definition of eq $6 \mathrm{c}$ is included in eq A1. From the quoted limits of $\Phi_{\mathrm{NP}}$ we can establish that $x$ ranges between 1 and 0.6 at lower limit.

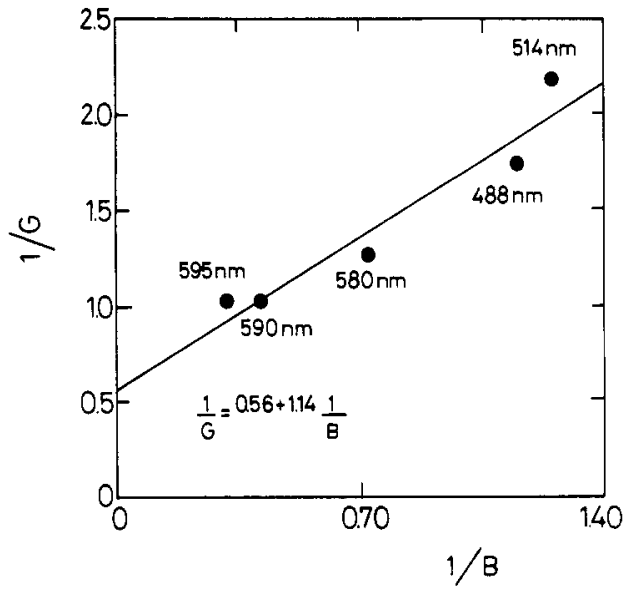

Figure 4. Plot of $1 / G$ vs $1 / B$ (see text).

TABLE II: Photophysical Parameters for MC540 Stable Ground State (N) and Photoisomer (P) in Ethanol $95 \%$ at $20^{\circ} \mathrm{C}$

\begin{tabular}{lll}
\hline \multicolumn{1}{c}{ parameter } & \multicolumn{1}{c}{$\mathrm{N}$} & \multicolumn{1}{c}{$\mathrm{P}$} \\
\hline$\sigma_{\max }(560 \mathrm{~nm}), \mathrm{cm}^{2}$ & $6.31 \times 10^{-16}$ & $4.74 \times 10^{-16 b}$ \\
$\epsilon_{\max }(560 \mathrm{~nm}), \mathrm{M}^{-1} \cdot \mathrm{cm}^{-1}$ & $165 \times 10^{3 a}$ & $125 \times 10^{3 b}$ \\
fluor lifetime, ps & $430^{a}$ & $230^{b, c}$ \\
fluor quantum yield & $0.17^{a}$ & $0.07^{b}$ \\
photoisomer quantum yield & $0.40^{b}$ & $0.20^{b}$ \\
thermal isomer rate const, $\mathrm{s}^{-1}$ & & $0.85^{a}$ \\
energy diff to $\mathrm{N}$ ground state, $\mathrm{kJ} \cdot \mathrm{mol}^{-1}$ & & $165^{b}$
\end{tabular}

${ }^{a}$ Values from ref $10 .{ }^{b}$ This work. ${ }^{c}$ Calculated from Strickler and Berg ${ }^{13}$ using 0.07 for the fluorescence quantum yield. See also text.

Flash photolysis results must be analyzed for $\Phi_{\mathrm{PN}} \neq 0$. In the Appendix, the common flash photolysis equations, obtained for complete conversion, are generalized in order to include the photoequilibrium. Absorbance difference spectra obtained either at low energy excitation $(\Delta A)$ or at saturation regime $\left(\Delta A_{\infty}\right)$ can be expressed in terms of $x$. The values of $\Phi_{\mathrm{NP}}, \Delta \epsilon$, and $\Phi_{\mathrm{PN}}$ as a function of $x$, calculated by using eqs 6 , are given in Table I.

To obtain $p$ and $f$ we have to use an iterative procedure. We begin with the $B$ values obtained from the assumption of $\Phi_{\mathrm{PN}}=$ $0(x=1)$ in order to obtain the first estimation of $p$ and $f$ from the slope and intercept of the representation of eq 5. The value of $p$ gives a better estimate of $x$. From the specific value of $x$, the parameters quoted in Table I can be calculated by using eqs 6 and a corresponding set of $B$ is obtained. From the plot of $1 / G$ vs $1 / B$ a new estimation of $p$ allows the procedure to be reproduced until two successive iterations render no difference in the value of $x$. The final values are plotted in Figure 4, from which

$$
p=0.49 \pm 0.17 \quad f=0.39 \pm 0.13
$$

From Table I, taking the latter value of $p$ as entry, we estimate $x=0.74 \pm 0.08$. This renders $\Phi_{\mathrm{NP}}=0.40 \pm 0.04$ from eq $6 \mathrm{a}$. $\Phi_{\mathrm{PN}}=0.20 \pm 0.04$ and $\Phi_{\mathrm{f}}{ }^{\mathrm{P}}=0.07 \pm 0.02$ are calculated from the definition of $p$ and $f$ considering the known value $\Phi_{\mathrm{f}}{ }^{\mathrm{N}}=0.17$.

The photophysical parameters of the photochromic system $\mathrm{P}, \mathrm{N}$ are quoted in Table II. The lifetime of ${ }^{1} \mathrm{P}$ (P fluorescence lifetime) was calculated from the formula of Strickler and Berg ${ }^{13}$ and $\Phi_{f}{ }^{P}$ $=0.07$. The value may be considered realistic on the basis of the good agreement obtained from the similar calculations for ${ }^{1} \mathrm{~N}$. From the value of $\Phi_{\mathrm{NP}}$, the energy gap $\Delta E_{\mathrm{P}}=165 \pm 25 \mathrm{~kJ} \cdot \mathrm{mol}^{-1}$ can be calculated by using previous optoacoustic results (see Appendix and ref 10 ).

Considering a model for the isomerization in which all the nonradiative deactivations (photoisomerizations and internal conversion) of excited singlet states, either ${ }^{1} \mathrm{~N}$ or ${ }^{1} \mathrm{P}$, take place from a common twisted state ${ }^{14}$ we obtain

$$
\alpha=\frac{\Phi_{\mathrm{NP}}}{1-\Phi_{\mathrm{f}}^{\mathrm{N}}} \text { and } 1-\alpha=\frac{\Phi_{\mathrm{PN}}}{1-\Phi_{\mathrm{f}}^{\mathrm{P}}}
$$


where $\alpha$ is related to the branching ratio defined by Rullière. ${ }^{14}$ Equation 8 does not hold for MC540 according to the results of Table II. In fact, $\Phi_{\mathrm{NP}} /\left(1-\Phi_{\mathrm{f}}{ }^{\mathrm{N}}\right)=0.48$ and $\Phi_{\mathrm{PN}} /\left(1-\Phi_{\mathrm{f}}{ }^{\mathrm{P}}\right)=$ 0.22 . This result indicates that the model cannot be applied to MC540; i.e., either ${ }^{1} \mathrm{~N}$ or ${ }^{1} \mathrm{P}$ or both of them deactivate through direct internal conversion. Regarding the values of $\Phi_{\mathrm{PN}}=0.20$ and $\Phi_{f}{ }^{P}=0.07$, we conclude that the main deactivation of ${ }^{1} P$ is internal conversion which can take place as a direct process or through a twisted state. A similar result was found for DODCI. $12,15,16$

Finally, we can obtain quantitative conclusions regarding the activation barrier in the excited singlet energy surface which is responsible for the temperature dependence of $\Phi_{\mathrm{f}} \mathrm{N}$ and $\Phi_{\mathrm{Np}} .{ }^{10} \mathrm{We}$ assume that all the processes competing with fluorescence take place from a twisted excited state $(t)$. Under this condition

$$
\tau_{\mathrm{f}}^{\mathrm{N}}=\left(k_{\mathrm{f}}^{\mathrm{N}}+k_{\mathrm{N} t}\right)^{-1}
$$

where $k_{\mathrm{f}} \mathrm{N}$ is the radiative rate constant of $\mathrm{N}$ and $k_{\mathrm{Nt}}$ the one corresponding to the passage to the twisted state. $k_{\mathrm{f}}^{\mathrm{N}}$ is assumed to be independent of temperature and has a value of $3.95 \times 10^{8}$ $\mathrm{s}^{-1}$ calculated from

$$
\Phi_{\mathrm{f}}{ }^{\mathrm{N}}=k_{\mathrm{f}}^{\mathrm{N}} \tau_{\mathrm{f}}^{\mathrm{N}}
$$

and the values of Table II. If we replace (9) in (10), we obtain

$$
k_{\mathrm{Nt}}=k_{\mathrm{f}} \mathrm{N}\left(\frac{1}{\Phi_{\mathrm{f}}^{\mathrm{N}}}-1\right)
$$

We can calculate the temperature dependence of $k_{\mathrm{Nt}}$ using eq 11 and the values of Table $I$ in ref 10 for $\Phi_{f}^{N}$ at temperatures lower than $300 \mathrm{~K}$. In this way, $23 \pm 1 \mathrm{~kJ} \cdot \mathrm{mol}^{-1}$ is obtained from an Arrhenius plot. This is the activation energy for the photoisomerization step. From the value of the photoisomerization rate constant $k_{\mathrm{NP}}=\Phi_{\mathrm{NP}} / \tau_{\mathrm{f}}^{\mathrm{N}}=0.40 \times(430 \mathrm{ps})^{-1}=9.30 \times 10^{8} \mathrm{~s}^{-1}$ at $20^{\circ} \mathrm{C}$ (see Table II), a preexponential factor of $1.2 \times 10^{13} \mathrm{~s}^{-1}$ is calculated.

The experimental activation energy, obtained under the assumption of a complete nonradiative deactivation via a twisted state, is equal to the energy barrier between ${ }^{1} \mathrm{~N}$ and " $t$ ". This model may not be realistic for ' $\mathrm{N}$, as was discussed above, and direct internal conversion may take place. In the latter case, the value of $23 \mathrm{~kJ} \cdot \mathrm{mol}^{-1}$ is a lower limit for the energy barrier.

\section{Conclusions}

The measurements performed in this work analyzed in conjunction with results from flash photolysis experiments provide a complete quantitative description of a photochromic system with very short lifetimes for excited states and complete overlap of ground-state absorption.

It must be pointed out that flash photolysis experiments need to be carefully interpreted in order to avoid erroneous results, particularly when high fluences are used in the excitation pulse.

Steady-state fluorescence is a very sensitive technique to study photochromic systems. It has the additional advantage that a maximum conversion limit can be reached with very low excited singlet state populations.

Acknowledgment. We are members of the Carrera del Investigador Cientifico from the Consejo Nacional de Investigaciones Cientificas y Ténicas of Argentina (CONICET). The work was performed under financial support from CONICET (Grants PID 3081500 and PIA E0919), the Stiftung Volkswagenwerk, and the Comisiôn de Investigaciones Cientificas de la Provincia de Buenos

(14) Rullière, C. Chem. Phys. Lett. 1976, 43, 303.

(15) Bilmes, G. M.; Braslavsky, S. E.; Tocho, J. O. J. Phys. Chem. 1988, 92,5958 .

(16) Rentsch, S.; Döpel, E.; Petrov, V. Appl. Phys. B 1988, 46, 357.
Aires (CICPBA). We thank Prof. Dr. Silvia E. Braslavsky for encouragement and helpful discussion.

\section{Appendix}

Flash Photolysis Equations under Photoequilibrium. We shall discuss some results of ref 10 and we shall generalize some common flash photolysis equations and apply them to a photochromic system.

From the application of the complete conversion method in flash photolysis, the difference absorption coefficient $\left(\Delta \epsilon=\epsilon_{\mathrm{P}}-\epsilon_{\mathrm{N}}\right)$ at $560 \mathrm{~nm}$ was calculated as $\Delta \epsilon_{560}=-30 \times 10^{3} \mathrm{M}^{-1} \cdot \mathrm{cm}^{-1}$ assuming that $P$ did not isomerize back to $N$ (i.e., $\Phi_{\mathrm{PN}}=0$ ). When this value was used to compute the isomerization quantum yield $\left(\Phi_{\mathrm{NP}}\right)$, a value of 0.54 was obtained.

On the other hand, assuming that $P$ cannot be higher in energy than ' $\mathrm{N}$ for an efficient photoisomerization, i.e., $\Delta E_{\mathrm{p}}$ as defined in Figure 3 is $210 \mathrm{~kJ} / \mathrm{mol}$ (the energy gap between $\mathrm{N}$ and ${ }^{1} \mathrm{~N}$ ) and taking into account the result $\Phi_{\mathrm{NP}} \Delta E_{\mathrm{P}}=67 \pm 6 \mathrm{~kJ} / \mathrm{mol}$ from LIOAS, $\Phi_{\mathrm{NP}}=0.32$ is obtained. The corresponding value for the difference absorption coefficient would be $\Delta \epsilon_{560}=-50 \times 10^{3}$ $\mathrm{M}^{-1} \cdot \mathrm{cm}^{-1}$. This limit would correspond to the maximum photoisomerization quantum yield from $P$ to $N\left(\Phi_{\mathrm{PN}}\right)$.

During the laser irradiation (pulse of $15 \mathrm{~ns}$ fwhm) we can integrate the kinetic equations derived from Figure 3 under the conditions of low absorbance and steady state for only ${ }^{1} \mathrm{~N}$ and ${ }^{1} \mathrm{P}$ (see eq 1$)$ and in the absence of flux $(w=0)$, neglecting the thermal decay $k$ from $P$ to $N$. The integration renders, for $[\mathrm{P}]_{f}$ the concentration of $\mathrm{P}$ at the end of the pulse of energy $E_{1}$, cross section $S$, and wavelength $\lambda$

$$
[\mathrm{P}]_{\mathrm{f}}=\frac{\epsilon_{\mathrm{N}} \Phi_{\mathrm{NP}}}{\epsilon_{\mathrm{P}} \Phi_{\mathrm{PN}}+\epsilon_{\mathrm{N}} \Phi_{\mathrm{NP}}} c_{0}\left(1-e^{-b E_{l}}\right)=x c_{0}\left(1-e^{-b E_{1}}\right)
$$

with

$$
b=2.303\left(\epsilon_{\mathrm{N}} \Phi_{\mathrm{NP}}+\epsilon_{\mathrm{P}} \Phi_{\mathrm{PN}}\right) \frac{\lambda}{h c S}
$$

$c_{0}$ is the total concentration, and $x$ is defined as the fraction of $c_{0}$ which is present as $P$ in the saturation-photostationary state ( $[\mathrm{P}]_{\infty}$ corresponds to $E_{1} \gg b^{-1}$ ). If $\Phi_{\mathrm{PN}}=0, x=1$ is the limit of the complete conversion. If $\Phi_{\mathrm{PN}} \neq 0$, then the measurement of $\Delta A_{\infty}$ (the absorbance difference at saturation) at a certain analyzing wavelength, $\lambda_{\alpha}$, is

$$
\Delta A_{\infty}\left(\lambda_{\alpha}\right)=\left(\epsilon_{\mathrm{P}}-\epsilon_{\mathrm{N}}\right)_{\lambda_{a}} l[\mathrm{P}]_{\infty}=\Delta \epsilon_{\lambda_{\alpha}} l x c_{0}
$$

from which

$$
\frac{\Delta A_{\infty}\left(\lambda_{\alpha}\right)}{l c_{0}}=\Delta \epsilon_{\lambda_{\alpha}} x=-30 \times 10^{9} \mathrm{M}^{-1} \cdot \mathrm{cm}^{-1} \quad \text { at } \lambda_{\alpha}=560 \mathrm{~nm}
$$

This value is an average of the measurements in ref $10 . \Phi_{\mathrm{NP}}$ can be expressed, as a function of the absorbance difference $(\Delta A)$ and absorbed dose $\left(I_{\alpha}\right)$, as

$$
\Phi_{\mathrm{NP}}=\frac{\Delta A\left(\lambda_{\alpha}\right)}{l \Delta \epsilon_{\lambda_{\alpha}} I_{\alpha}}
$$

When $\Delta \epsilon_{\lambda_{\alpha}}$ from eq $A 3$ is replaced in eq $A 4$, we obtain eq $6 a$ of the text:

$$
\Phi_{\mathrm{NP}}=\frac{\Delta A\left(\lambda_{\alpha}\right) c_{0}}{\Delta A_{\infty}\left(\lambda_{\alpha}\right) I_{\alpha}} x=\Phi_{\mathrm{NP}}^{\max ^{2}} x=0.54 x
$$

We now have the equations to obtain the values of Table I. Equation $A 5$ gives $\Phi_{\mathrm{NP}}$ in terms of $x$. Equation $\mathrm{A} 3$, the definition of $\Delta \epsilon_{\lambda_{\alpha}}$, and eq Al were used to calculate $\Delta \epsilon(\lambda), \epsilon_{\mathrm{P}}(\lambda)$, and $\Phi_{\mathrm{PN}}$, respectively.

Registry No. MC540, 62796-23-0. 